\title{
REVIEW
}

\section{Robotic assisted andrological surgery}

\author{
Sijo J Parekattil and Ahmet Gudeloglu
}

The introduction of the operative microscope for andrological surgery in the 1970s provided enhanced magnification and accuracy, unparalleled to any previous visual loop or magnification techniques. This technology revolutionized techniques for microsurgery in andrology. Today, we may be on the verge of a second such revolution by the incorporation of robotic assisted platforms for microsurgery in andrology. Robotic assisted microsurgery is being utilized to a greater degree in andrology and a number of other microsurgical fields, such as ophthalmology, hand surgery, plastics and reconstructive surgery. The potential advantages of robotic assisted platforms include elimination of tremor, improved stability, surgeon ergonomics, scalability of motion, multi-input visual interphases with up to three simultaneous visual views, enhanced magnification, and the ability to manipulate three surgical instruments and cameras simultaneously. This review paper begins with the historical development of robotic microsurgery. It then provides an in-depth presentation of the technique and outcomes of common robotic microsurgical andrological procedures, such as vasectomy reversal, subinguinal varicocelectomy, targeted spermatic cord denervation (for chronic orchialgia) and robotic assisted microsurgical testicular sperm extraction (microTESE).

Asian Journal of Andrology (2013) 15, 67-74; doi:10.1038/aja.2012.131; published online 17 December 2012

Keywords: andrological surgery; male infertility; microsurgery; robotics; robotic assisted microsurgery; robotic vasectomy reversal; vasectomy reversal

\section{INTRODUCTION AND HISTORICAL PERSPECTIVE}

Karel Capek (a Czech writer) and Josef Capek (a painter, Karel's older brother) initially coined the term 'robot' to describe artificial workers in a Czech play in the 1920s. ${ }^{1}$ In the 1960s, robots were developed for industrial applications and the expansion of robotics has continued from military to medical applications. The current, most widely utilized medical surgical robotic platform (da Vinci, Intuitive Surgical, Sunnyvale, CA, USA) was launched in 1999 and has had several upgrades and revisions to extend its use for a wider range of medical applications. The use of robotics in urology is widespread, from its initial use for radical prostatectomy in the early 2000s to partial nephrectomy and radical cystectomy currently., ${ }^{2,3}$

Even though the advantages of enhanced magnification, elimination of tremor and scaling of motion really do help in minimally invasive or laparoscopic surgery, it is intuitive to imagine that these benefits would only be further enhanced when applying this platform for delicate microsurgical procedures. This is what led to the initial use of robotics for microsurgery.

Kuang et al. ${ }^{4}$ performed some initial work in robotic assisted surgery in an ex vivo vasovasostomy model in 2004. Schiff et al..$^{5}$ then performed the first randomized prospective study comparing robotic assisted vasovasostomy (RAVV) and pure microsurgical vasovasostomy (MVV). This ground-breaking work clearly showed advantages of robotic assistance in terms of decreasing operative duration and decreasing sperm granuloma formation at the anastomosis. In 2004, Fleming et al. ${ }^{6}$ also reported the first bilateral RAVV on two patients with excellent patency results. In 2008, Corcione et al. ${ }^{7}$ and Shu et al. ${ }^{8}$ described the first robotic assisted subinguinal microsurgical varicocelectomy (RAVx). In 2008, our group introduced the use of robotic assistance for targeted microsurgical denervation of the spermatic cord (RMDSC) for patients with chronic groin or testicular pain. ${ }^{9,10}$

The initial inertia in the use of robotics in microsurgery has primarily been a lack of access to the robot by microsurgeons and the 10-fold increase in cost of the equipment in comparison to standard microsurgical equipment. However, our group has recently published a larger volume study comparing robotic to pure microsurgical vasectomy reversal and have shown that with increased volume, enhanced surgical efficiency, eliminated need for a skilled microsurgical assistant, and decreased operative time, these procedure can be performed at a cost similar to or even less than standard microsurgical procedures. ${ }^{11}$

Our group has now performed over 800 robotic assisted microsurgical procedures and this is our preferred method of microsurgery. This article will provide a detailed presentation of the surgical technique and outcomes of robotic assisted microsurgical urological procedures, such as vasectomy reversal, varicocelectomy, targeted denervation of the spermatic cord for chronic testicular pain/orchialgia (CO), robotic microsurgical testicular sperm extraction (ROTESE) and microvascular anastomosis for inadvertent testicular artery injury during microsurgical procedures.

\section{ROBOTIC ASSISTED MICROSURGICAL VASECTOMY REVERSAL}

Vasectomy has become one of the most reliable family-planning methods currently available, because of its simplicity and effectiveness with a low morbidity rate. An estimated 40-60 million men worldwide 
rely on this method of contraception. ${ }^{12-14}$ Vasectomy is one of the most common urological procedures performed in the United States. ${ }^{15}$ About $2 \%-6 \%$ of these men undergo a reversal operation within the first 10 years after the vasectomy, because of a desire to become fertile again. ${ }^{14,16}$ Although complications from vasectomy are rare, up to $10 \%$ of men after vasectomy might suffer chronic postvasectomy testicular pain affecting the quality of life. Congestive epididymitis is one possible mechanism for this type of pain and vasectomy reversal has been shown to be one option for these men. ${ }^{17}$

Vasectomy reversal is a technically demanding procedure. The procedure was initially described in 1919 and this macroscopic technique achieved only $60 \%$ patency rates until the introduction of the operating microscope in the 1970 s. $^{18,19}$ Silber et al. ${ }^{20,21}$ introduced the microscope into clinical urology and improved patency rates from approximately $60 \%-94 \%$ over a 4000 cases experience in 30 years. The use of the operative microscope for vasovasostomy to achieve greater fertility rates and vas patency has become the standard in Practice Committee of American Society for Reproductive Medicine. ${ }^{22}$ However, achieving excellent outcomes requires extensive microsurgical laboratory training and clinical experience. ${ }^{23}$ A skilled microsurgical assistant is another necessity for these complex microsurgical procedures.

Kuang et $a l .{ }^{4}$ performed one of the initial robotic microsurgical studies that compared RAVV to the pure MVV on an ex vivo vasovasostomy model. They found that there was a learning curve associated with RAVV-initially, the RAVV cases where longer and the instruments were more likely to break sutures and needles bends compared to MVV. However, the RAVV operative duration rapidly decreased with each with consecutive procedure from 104 to $62 \mathrm{~min}$ in as few as five cases. This study illustrates the potential of the robot to shorten the learning curve for surgeons in microsurgical skills training.

Schiff et al. ${ }^{5}$ then followed up with the first randomized control trial comparing standard MVV and vasoepididymostomy to robot assisted procedures in a rat model. Although the patency rates were $100 \%$ in RAVV and robotic assisted vasoepididymostomy (RAVE) groups and $90 \%$ in MVV and microsurgical vasoepididymostomy (MVE) groups, this did not reach statistical significance due to the small sample size. However, the sperm granuloma formation rate at the anastomosis (the rats were killed a few weeks later to assess for anastomosis healing) was significantly lower in the RAVV group at $27 \%$ verses $70 \%$ in the MVV group ( $P=0.001)$. According to the authors, this result was likely due to the improved precision of suture placement with the robotic instruments, resulting in a more watertight anastomosis. Operative duration for RAVV was also found to be significantly faster than MVV (68.5 $\min v s .102 .5 \mathrm{~min}, P=0.002$ ).

Fleming et $a .^{6}$ then demonstrated the first human RAVV procedures in 2004 in two patients with 100\% patency. They highlighted the shorter learning curve compared to traditional microsurgical techniques and the possibility of greater ease and precision of suture placement.

Our group has recently published a prospective database cohort study comparing 110 robotic reversals (66 cases bilateral RAVV, 44 cases RAVE on at least one side) to 45 pure microscopic reversals (28 cases bilateral MVV and 17 cases MVE on at least one side). ${ }^{11}$ Median clinical follow-up was 17 months (range 1-52 months). Median duration from vasectomy in the RAVV group was 7 years (range 1-21 years) and 6.5 years (range $1-19$ years) in the MVV group $(P=0.3)$. Median age of the patients in the RAVV group was 41 and 39 years in the MVV group $(P=0.4)$. A significantly better patency rate of $96 \%$ was achieved in the RAVV cases versus $80 \%$ in MVV $(P=0.02)$.
Pregnancy rates (within one year post-operatively) did not differ significantly for the two groups: $65 \%$ for the RAVV and 55\% for the MVV. Operative duration (skin to skin) started at 150 to $180 \mathrm{~min}$ initially for the first 10 cases for RAVV. However, the median operative duration for RAVV overall was significantly decreased at $97 \mathrm{~min}$ (range 40-180 min) compared with MVV at $120 \mathrm{~min}$ (range 60$180 \mathrm{~min})(P=0.0003)$. RAVE at $120 \mathrm{~min}$ (range 60-180 min) was significantly faster than MVE at 150 minutes (range 120-240 min) $(P=0.0008)$. This work helps to consolidate the evidence from the earlier studies.

\section{Robotic surgical platform and setup}

Intuitive Surgical (Sunnyvale, CA, USA) offers a four-arm da Vinci type Si robotic system with high definition digital visual magnification (up to $\times 10-\times 15$ ). The left and right arms are loaded with Black Diamond Micro Needle Drivers (Intuitive Surgical, Inc., Sunnyvale, CA, USA). The additional fourth arm provides the microsurgeon with one additional tool such as Micro Potts Scissors (Intuitive Surgical, Inc.).

Our team has developed a unique five-arm approach for robotic microsurgical procedures (Figure 1 ). A $\times 16-\times 18$ optical high magnification lens camera system is attached to the patient table. The VITOM (Karl Storz Inc., Tuttlingen, Germany) camera system is held by a nitrogen powered fifth arm (Point setter arm; Karl Storz Inc., Tuttlingen, Germany). The real-time video images from the VITOM are incorporated into the surgeon console view utilizing the TilePro (Intuitive Surgical) software system to provide simultaneous real-time images to the microsurgeon (Figure 2). The TilePro software allows for displaying up to three simultaneous real-time images from any source. Figure 2 illustrates the cockpit view of the microsurgeon in the surgeon console: (i) the da Vinci Si 3D HD camera view in the middle (the main image), (ii) the VITOM $\times 16-\times 18$ optical magnification camera view and (iii) a $\times 40-\times 100$ optical microscopic view from the intra-operative andrology laboratory microscope (Nikon Inc., Tokyo, Japan).

This tri-view in the robotic console allows the surgeon to use the da Vinci camera for the overall tissue and suture handling at a medium zoom and simultaneously provides the VITOM $\times 16-\times 18$ magnification view to see fine ultrastructural detail at high zoom. In standard microscopic microsurgery, due to the limited depth of focus, the microscope has to be zoomed in or out for different components of the procedure. The use of this tri-view system improves operative efficiency by obviating the need to zoom in and out during microsurgical procedures, since the surgeon is essentially able to see the field simultaneously at two different focal lengths.

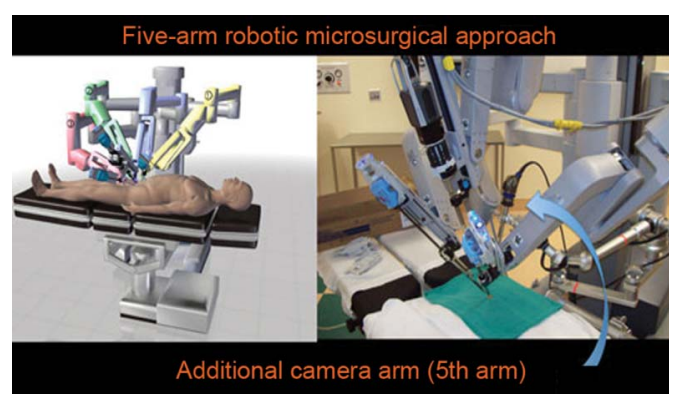

Figure 1 Five-arm approach: utilizes the Point-Setter nitrogen powered arm and the VITOM high magnification lens providing $\times 16-\times 18$ optical magnification (Karl Storz Inc., Tuttlingen, Germany). This provides the surgeon with an additional camera view in the surgeon console. 


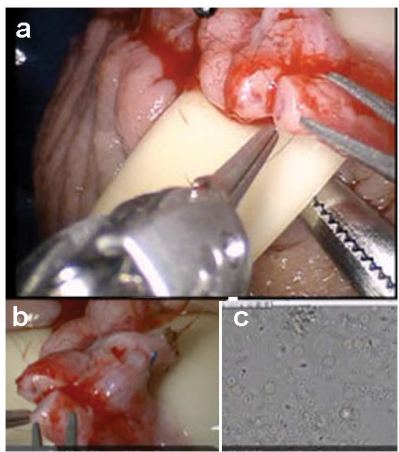

Figure 2 A cockpit tri-view in the surgeon console: (a) main view from the da Vinci Si 3D HD camera (Intuitive Surgical Inc., Sunnyvale, CA, USA), (b) view from the VITOM $\times 16-\times 18$ optical magnification camera lens system (Karl Storz Inc., Tuttlingen, Germany) and (c) view from the $\times 40-\times 100$ optical microscopic view from the andrology laboratory microscope (Nikon Inc., Tokyo, Japan).

The ability to also simultaneously see the fluid that the andrologist is assessing for sperm provides a significant improvement in operative efficiency by enhancing the real-time communication between the microsurgeon and the lab staff (this alone has saved 15-20 min on procedures since the surgeon is able to communicate and visualize the fluid real-time with the andrologist while operating). The surgeon no longer has to leave the operative field to check this fluid-this image is sent right into the surgeon console. The general operative setup for the robotic microsurgical procedures is as illustrated in Figure 3.

This could be argued as primarily being more of an issue of having personnel to examine fluid rather than a $\$ 1$ million piece of equipment to perform this function. Indeed, a competent circulating nurse or student, a digital camera, and monitor could accomplish the same function. Alternatively, someone could just say, 'I see sperm.' However, this feature becomes increasingly beneficial such as, in microTESE cases where continuous sampling of the testicular tissues can be visualized by the surgeon without surgical interruption. As the cost of such technology falls (with competing platforms becoming available), this may become less of a counter-issue.

\section{Surgical technique for RAVV technique}

Patient positioning and preparation of the two ends of the vas or the epididymis are similar to the standard microsurgical fashion. The

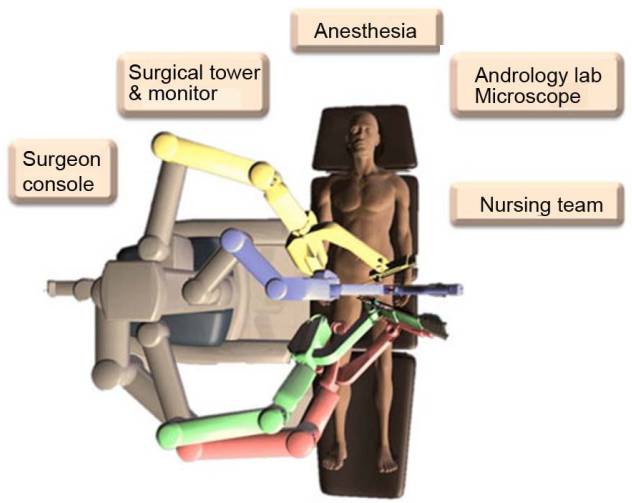

60" High definition viewing monitor

Figure 3 General layout of the operative room equipment for robotic assisted microsurgical procedures. distal vas (away from the testicle) is dissected to allow a tension-free anastomosis to the proximal vas or epididymis. The proximal vas is carefully transected with a \#11 blade. Efflux from the lumen is expressed and collected on a glass slide. Phase contrast microscopy (andrology lab microscope) is used to assess for the presence of sperm on the slides. If there is any sperm found and the efflux is copious, clear or milky, then a RAVV is performed. If the efflux has no sperm and is thick and pasty, then a RAVE is performed.

The distal end of the vas is now transected. The two clean ends of the vas are now approximated to each other to confirm a tension free anastomosis. The two ends of the vas are placed over a $1 / 4^{\prime \prime}$ Penrose drain. The adventitia from either end of the vasa is now secured together with a 3-0 prolene suture to create a tension free anastomosis. The robot is now positioned to perform the MVV. The right side vasovasostomy is generally performed first. Black diamond microforceps are loaded on the right and left robot arms. The zero degree camera lens is loaded into the robot camera arm. The micro-Potts scissors are loaded on the 4 th robot arm. The assistant irrigates the field with saline using a $10-\mathrm{ml}$ syringe with an 18-gauge angiocatheter tip.

The assistant now passes the 9-0 nylon suture that is kept in its inner foam packaging to the edge of the surgical field. The suture is grasped by the surgeon using the black diamond right hand grasper and cut to about 2 inches length using the micro Potts scissors (left hand fourth arm). The 9-0 nylon suture is held and manipulated using the black diamond forceps in both left and right arms as needle drivers. The posterior muscularis layer of the two ends of the vas is now approximated (Figure 4a). The suture is cut using the micro Potts scissors (fourth arm).
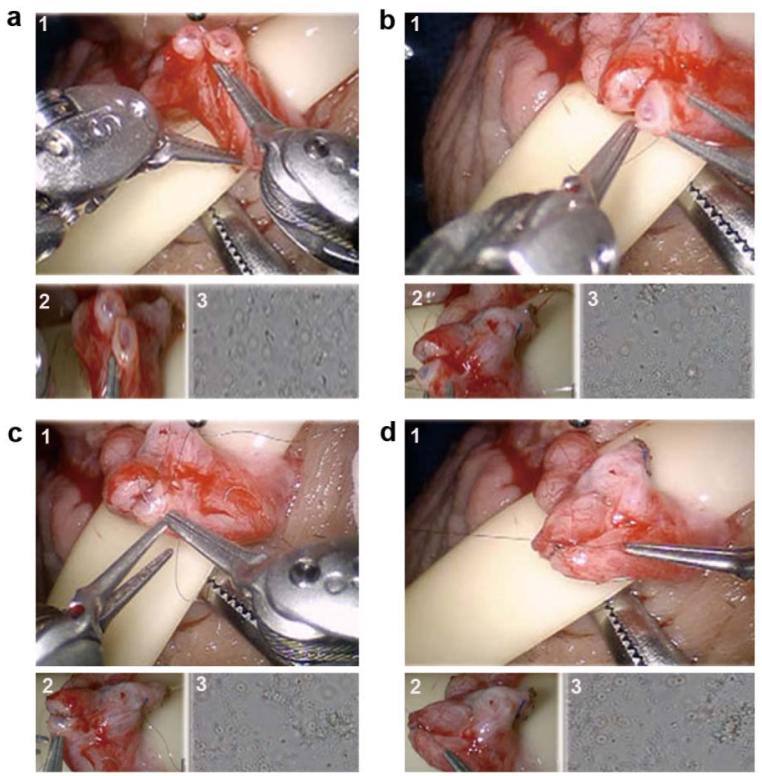

Figure 4 Surgical technique for robotic assisted vasovasostomy technique. (a) Suture placement for posterior vasal muscularis layer anastomosis with 9-0 nylon. (b) Suture placement for posterior vasal mucosal lumen anastomosis with double armed 10-0 nylon. (c) Suture placement for anterior vasal mucosal lumen anastomosis with double armed 10-0 nylon. (d) Suture placement for anterior vasal muscularis layer anastomosis with 9-0 nylon. For all above: (1) Main view from the da Vinci Si 3D HD camera (Intuitive Surgical, Sunnyvale, CA), (2) View from the VITOM $\times 16-\times 18$ optical magnification camera lens system (Karl Storz Inc., Tuttlingen, Germany) and (3) View from the $\times 40-\times 100$ optical microscopic view from the andrology laboratory microscope (Nikon Inc., Tokyo, Japan). 
Two or three double-armed 10-0 nylon sutures are now placed to re- anastomose the posterior mucosal lumen of the vas (Figure $\mathbf{4 b}$ ). The sutures are placed inside out to ensure good mucosal approximation. All posterior sutures are placed before they are tied.

Three double-armed 10-0 nylon sutures are now used to close the anterior mucosal lumen of the vas (Figure 4c). Five to six 9-0 nylon sutures are used to approximate the anterior muscularis layer of the vas (Figure 4d). The Penrose drain is gently removed from under the repair. The vas is placed back into the scrotal cavity. The same procedure is now performed on the contralateral left side by repositioning the robotic arms to the left scrotum.

The dartos layer is closed using a running 3-0 chromic suture. The skin is closed using a 5-0 vicryl running suture. Bacitracin ointment is applied over the incision. Fluff dressing with athletic scrotal support is applied. An ice pack is carefully applied to the scrotum in the recovery room.

\section{Surgical technique for RAVE}

The robotic vasoepididymostomy procedure starts from above if there is no sperm in the fluid from the proximal vas and the fluid is thick and pasty. The scrotal incision is enlarged by another $1-2 \mathrm{~cm}$ inferiorly. The testicle is delivered and the tunica is incised to expose the epididymis. The adventitial layer of the epididymis is incised above the level of epididymal obstruction (blue/grey zone with dilated epididymal tubules above this area). A 3-0 Prolene suture is utilized to approximate the tunica of the epididymis to the adventitia of the vas to create a tension-free anastomosis. The robot is now positioned to perform the MVE as described earlier. The black diamond microforceps are loaded on the right and left robot arms. The zero degree camera lens is loaded on the robot camera arm. An ophthalmologic micro blade is held in the fourth arm with black diamond micro forceps or a potts scissor may be used in the fourth arm. Two 10-0 nylon double-armed suture needles are placed longitudinally through a single epididymal tubule to expose the tubule (Figure 5a). This tubule is then incised longitudinally using the micro blade between the two suture needles to create a lumen in the tubule. Alternatively, the tubule may be incised with a Potts scissor in the fourth robotic arm. The fluid is then aspirated (Figure 5b) and then examined under a separate phase contrast microscope for the presence of sperm (andrology lab microscope).

The two double armed 10-0 nylon needles in the epididymal tubule are advanced through and then all four of the needles are brought inside out on the vas mucosal lumen to involute the epididymal tubule lumen into the vas lumen (Figure 5c). Five to six 9-0 nylon sutures are placed circumferentially to approximate the muscularis of the vas to the adventitia of the epididymal tubule (Figure 5d). The testicle and anastomosis are carefully delivered back into the scrotum. The dartos layer is closed using a running 3-0 chromic suture. The skin is closed using a 4-0 chromic running suture. Bacitracin ointment is applied over the incision. Fluff dressing with athletic scrotal support is applied. An ice pack is carefully applied to the scrotum in the recovery room.

\section{Robotic assisted microsurgical vasectomy reversal experience}

Our group has an institutional review board approved prospective database protocol to capture date prospectively on all our patients. Between July 2007 and June 2012, 117 robotic assisted vasectomy reversals were performed (71 bilateral RAVV, 46 at least one side RAVE) by a single fellowship-trained microsurgeon. The majority of these patients were undergoing the reversal to regain their fertility. Thirteen of these patients underwent the reversal, since they were suffering from congestive type
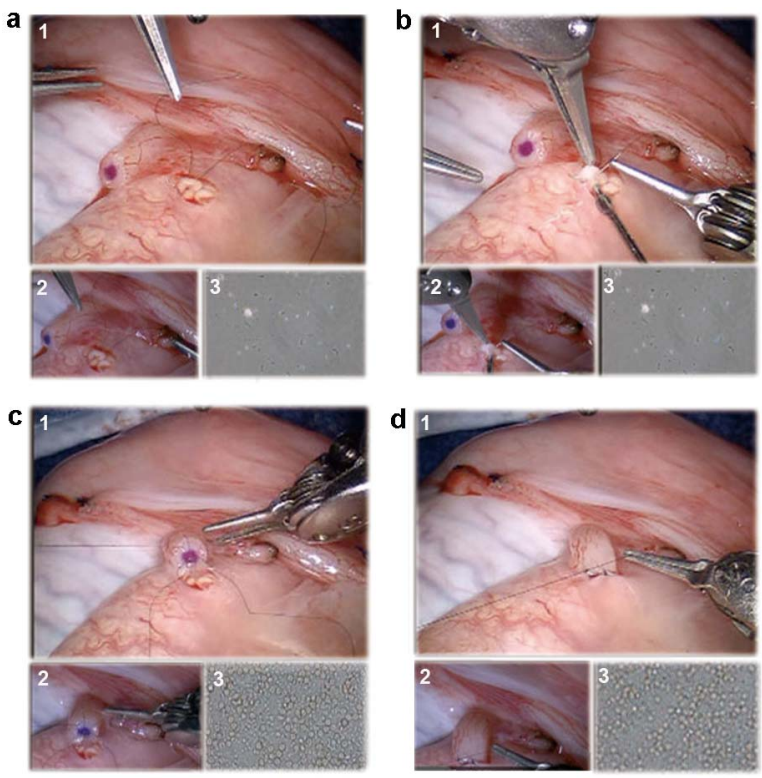

Figure 5 Surgical technique for robotic assisted vasoepididymostomy. (a) Two 10-0 nylon double-armed suture needles are placed longitudinally through a single epididymal tubule to expose the tubule. (b) Incision of the epididymal tubule with Potts scissors and aspiration of the epididymal fluid for microscopic examination. (c) All four needles of two double-armed 10-0 are brought inside out on the vas mucosal lumen to involute the epididymal tubule lumen into the vas lumen. (d) Five to six 9-0 nylon suture placement for vasal adventitia to epididymal tunica layer approximation. For all above: (1) Main view from the da Vinci Si 3D HD camera (Intuitive Surgical, Sunnyvale, CA, USA), (2) View from the VITOM $\times 16-\times 18$ optical magnification camera lens system (Karl Storz Inc., Tuttlingen, Germany) and (3) View from the $\times 40-\times 100$ optical microscopic view from the andrology laboratory microscope (Nikon Inc., Tokyo, Japan).

chronic post-vasectomy testicular pain (pain for at least one year after failing conservative treatment options). The median patient age was 42 years, and median duration from vasectomy was 7.5 years for RAVV and 11 years for RAVE. The median OR setup duration was $25 \mathrm{~min}$ and the median robotic microsurgical operative duration was $120 \mathrm{~min}$ for RAVV and $150 \mathrm{~min}$ for RAVE procedures. The median follow-up was 17 months. Patency rates ( $>1$ million motile sperm per ejaculate) were $97 \%$ in the RAVV group and $61 \%$ in the RAVE group. Pain relief was achieved in $92 \%$ of the patients who underwent RAVV for postvasectomy chronic testicular pain.

The robotic reversals initially had a longer operative duration (150$180 \mathrm{~min}$ ) than our current robotic procedures (usually 120-150 min depending on whether vasoepididymostomy is needed). There was an additional 30-60 min to prepare the robot at the beginning of the case. This extra time significantly decreased with experience as the operating room staff became more familiar with our setup. The duration of preparation for the robot (prior to the case) is routinely about 2025 min now (this is similar to the time the staff takes to prepare the microscope for pure microsurgical cases). This learning curve in the initial robotic cases was also observed by Kuang et al. ${ }^{4}$ Some centers may argue that 20-25 min to set up a microscope is excessively long. However, the purpose of this prospective outcomes database study was to compare a single center experience with both modalities and it appears that at our center, preparing the microscope or robot takes our OR staff a similar amount of time.

Based on the analysis of this large robotic assisted microsurgical vasectomy reversal series, the procedure appears to be safe and feasible. This series of RAVV cases presented in this study include all the 
robotic cases that the single surgeon has performed from the very first case. This surgeon who has completed dual fellowship training in microsurgery and robotics is still very early in his surgical career. What is interesting in this series is that the patency outcomes closely match those of microsurgeons who have performed several hundred MVV cases. $^{21}$ The data suggest that the robotics platform may improve or shorten the learning curve in terms of achieving better outcomes sooner. The patency outcomes in the MVV group were lower than expected published reports from mature series. However, the purpose of this prospective database study was to honestly report all outcomes from the surgeon's very first reversal cases and is presented to illustrate that robotic assistance did not produce inferior outcomes to standard microsurgery. It is likely that with experience, robotic outcomes are likely to be similar to an experienced microsurgeons' outcomes. In this particular case, robotic assistance appeared to help this surgeon's reversal outcomes.

The microscopic and robotic assisted cases were similarly performed temporally initially for the first two years, then more robotic cases were performed exclusively. This may explain some of the poorer initial outcomes in the pure microscopic group compared to the robotic assisted group due to the surgeons learning curve.

\section{ROBOTIC ASSISTED TARGETED MICROSURGICAL DENERVATION OF THE SPERMATIC CORD}

$\mathrm{CO}$ is defined as intermittent or constant, unilateral or bilateral testicular pain lasting more than 3 months. ${ }^{24,25}$ It has been shown that the prevalence of CO may occur in up to $33 \%$ of men after vasectomy and up to $43 \%$ of men after inguinal hernia repair. ${ }^{26,27}$ Pain can also occur after a sports injury to the groin area, abdominal surgery or any type of irritation of the ilioinguinal and/or genitofemoral nerves. Not all men with pain that causes them to seek medical attention, but it is estimated that this condition may affects up to 100000 men annually. $9,24,25,28,29$

The specific cause for CO is not well understood. The genitofemoral and ilioinguinal nerves provide innervation to the spermatic cord and testicle, and any type or hypersensitivity or irritation of these nerves may lead to CO. Any type of injury, such as infection, trauma, torsion, varicocele, hydrocele, scrotal surgery (most commonly vasectomy) or inguinal hernia repair, could trigger an inflammatory response in the peripheral nerve fibers in this area. It has also been shown that the peripheral immune response plays a pivotal role in the pathophysiology of peripheral neuropathic pain. ${ }^{30}$ Our group has performed specific pathology and anatomical studies and have identified specific abnormal nerve fibers (fibers with Wallerian degeneration) within the spermatic cord in patients who have chronic groin pain. ${ }^{31}$

This pain can be debilitating, thereby preventing the affected individual from performing simple tasks or even holding a job. Initially, non-surgical treatments are attempted: antibiotics, anti-inflammatory medications, analgesics, acupuncture, and physical therapy. When these approaches have failed, targeted nerve cord blocks may be attempted and if patients seem to respond to this, they may then be eligible to try denervation of the spermatic cord.

In the past, orchiectomy was usually performed for patients with intractable CO. However, the success rates after such procedures ranged only in the $20 \%-50 \%$ range and there was a risk of persistent phantom pain. Thus, Devine and Schellhammer ${ }^{32}$ first described microsurgical denervation of the spermatic cord (MDSC) as the first organ sparing technique for CO in 1978. This minimally invasive outpatient technique was further developed by Levine ${ }^{28}$ with an approximately $70 \%$ success rate in eliminating pain in these patients. This procedure entails the careful dissection and ligation of nerves and the bulk of the spermatic cord while preserving the testicular artery and some lymphatic channels along the spermatic cord.

Our team has further refined this technique based on the previously mentioned nerve mapping studies. ${ }^{31}$ We found that there were 31 reproducible small diameter $(<1 \mathrm{~mm})$ nerve fibers within the spermatic cord per patient. Eighty-four percent of orchialgia patients were found to have Wallerian degeneration in at least one or more of these nerves. Only $20 \%$ of patients had Wallerian degeneration in the control group - group of patient who had no pain, but were having groin surgery for other reason-such as testicular tumor $(P=0.0008)$. There were three primary locations for these abnormal nerves, listed in decreasing order of nerve density: (i) the cremasteric muscle fibers (19 nerves/patient), (ii) the peri-vasal tissues and vasal sheath (9 nerves/patient), and (iii) the posterior lipomatous/posterior-arterial tissues ( 3 nerves/patient). The nerve distribution found in the above study was then anatomically confirmed on separate cadaver dissections as well. We have now modified the denervation procedure to a targeted protocol where we only ligate three specific areas in the spermatic cord: (i) the cremasteric muscle layer around the cord, (ii) the peri-vasal tissues around the vas deferens and (iii) the posterior lipomatous and posterior peri-arterial tissues. We have also incorporated the use of the robotic platform to assist in this targeted microsurgical denervation technique. ${ }^{9}$

Since the peri-vasal tissue has the highest nerve density in the cord and it is difficult to fully ligate all this tissue while preserving the vas deferens and the vasa vasorum, we have explored the use of hydrodissection to help in selectively ligating nerve fibers around the vas while preserving the vessels. ${ }^{33}$ Gudeloglu et al..$^{33}$ have shown in this study in a rat model, that hydrodissection of the vas deferens at $87 \mathrm{psi}$ after the MDSC procedure significantly decreases the residual nerve fiber count on the vas without compromising blood flow or inducing vascular injury. Thus, we have incorporated hydrodissection of the vas deferens as a component of the RMDSC procedure.

Return or persistence of pain after MDSC of the spermatic cord for $\mathrm{CO}$ is quite disappointing for patients. One cause for this phenomenon could be neuroma formation or irritation of the ligated ends of the nerve fibers in the spermatic cord. Neuroprotective wraps have been safely and successfully utilized in peripheral nerve repair procedures to minimize such neuroma or scar formation. Thus, our group performed a study to evaluate the impact of a neuroprotective wrap placed around the spermatic cord after the denervation procedures. ${ }^{34}$ This was a prospective database cohort trial onsix patients who underwent bilateral RMDSC. A neuroprotective wrap (bio-inert matrix derived from porcine gut: Axoguard; Axogen Inc., Gainesville, FL, USA) was placed around the spermatic cord on one randomly selected side of each patient after completion of the denervation procedure. The contra-lateral side (with no wrap) was the control for each patient. Pain was assessed preoperatively and post-operatively at 1, 3, 6 and 12 months using an externally validated pain impact questionnaire (PIQ6; QualityMetric Inc., Lincoln, RI, USA). Median PIQ-6 scores on the wrap side were: $52,40,50$ and 59 at $1,3,6$ and 12 months postoperatively respectively. The median scores on the non-wrap side were: 59, 56, 60 and 68 respectively. The median pain scores on the side with the wrap were significantly less than the non-wrap side (a score change of 5 is significant: $P=0.05$ ). This study despite its small sample size indicated a possible benefit to using a neuroprotective wrap around the spermatic cord after denervation procedures for CO. Thus, we have also incorporated wrapping the cord after RMDSC with this material. 
Surgical technique for robotic assisted microsurgical denervation of the spermatic cord

The patient position and the initial steps of bringing up of the spermatic cord to the surface are similar to the standard MDSC procedure. A 1- to 2-cm transverse incision is made in the subinguinal area. The incision is carried down until the spermatic cord is reached. The spermatic cord is brought up to the surface. Posterior, medial and lateral dissection and cauterization are performed outside the cord to ligate branches of the ilioinguinal nerve and genitofemoral nerve in this area (near the lateral edge of the pubic symphysis).

The robotic microsurgical platform is now brought in to perform targeted MDSC. The right, left and the fourth robot arms are loaded with the Black Diamond microforceps, microbipolar grasper and curved monopolar scissors, respectively. The zero degree camera lens is loaded on the robot camera arm. The VITOM camera is also positioned to provide an additional magnified view of the cord.

The robotic phase of the technique starts with carefully cauterization and ligation of the cremasteric muscle layer. The intraoperative micro-Doppler (Vascular Technology Inc., Nashua, NH, USA) is employed for localizing the arteries (Figure 6a). With the aid of this device, all testicular arteries, veins and lymphatics are preserved. The peri-vasal sheath and the posterior lipomatous components are then carefully cauterized and ligated (Figure 6b). Residual nerve fibers around the vas deferens are ablated utilizing the ERBEJET2 hydrodissector tool (ERBE Inc., Atlanta, GA, USA). Figure 6c shows the
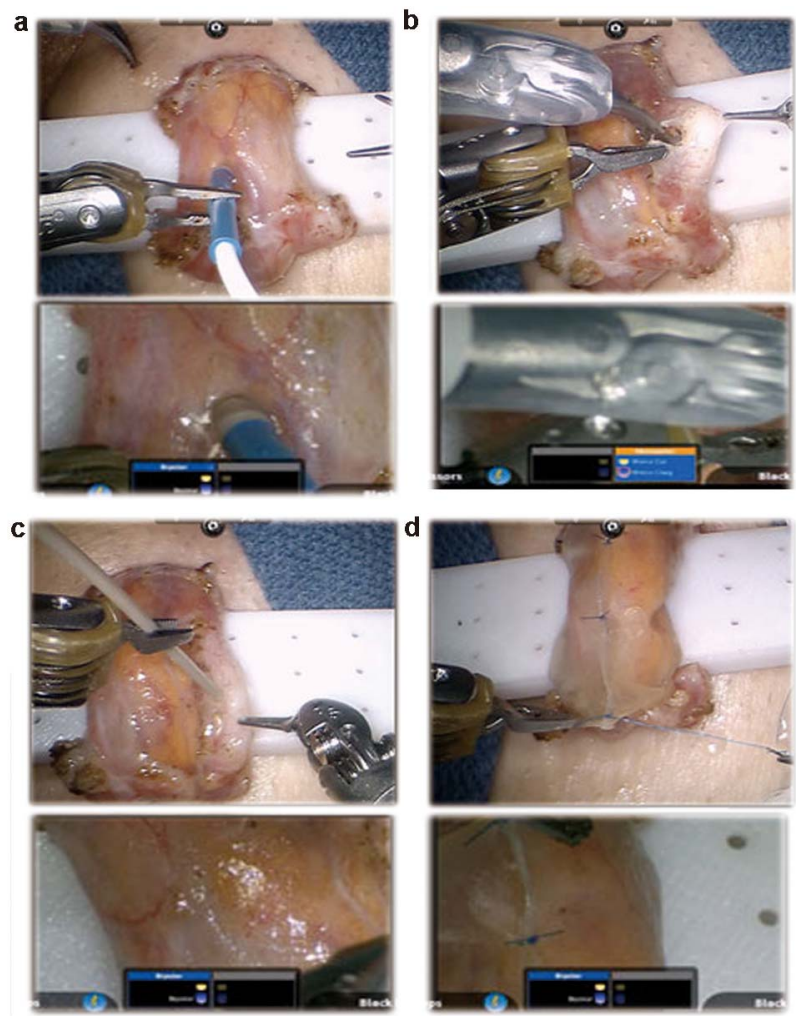

Figure 6 Surgical technique for robotic assisted microsurgical denervation of the spermatic cord. (a) The locations of testicular arteries are being determined utilizing the VTI (Vascular Technology Inc., Nashua, NH, USA) micro-Doppler probe. (b) Perivasal nerve fibers are cauterized. (c) Hydrodissection of residual pain fibers using the ERBEJET2 hydro-dissector probe (ERBE Inc., Atlanta, GA, USA). (d) Wrapping of the spermatic cord with Axoguard neuro-protective wrap (Axogen Inc., Gainesville, FL, USA) to prevent neuroma formation and prevent irritation or scarring at ligated nerve ends. hydrodissection of the vas deferens to ligate any small residual nerve fibers after the peri-vasal tissues are ligated with micro-cautery. The cord is then wrapped with the Axoguard (Axogen Inc., Gainesville, FL, USA) bio-inert wrap to prevent neuroma formation and irritation of ligated nerve ends and then loosely secured using 6-0 prolene interrupted sutures (Figure 6d). The cord is released back into the wound, and the wound is then closed.

\section{Targeted robotic assisted microsurgical denervation of the} spermatic cord experience

Our group has established an institutional review board approved prospective outcomes database to follow all these patients. Patients who had CO for more than 3 months and failed standard conservative treatment options were enrolled in the study. Between October 8 and June 12, 2012, 401 RMDSC procedures were performed. Pain was assessed utilizing a standardized validated pain assessment tool; PIQ6 (QualityMetric Inc., Lincoln, RI, USA). Pain scores and physical exam were performed preoperatively and then postoperatively at 1, 3, 6, 9 and 12 months. Median follow up was 23 months (1-44 months).

Eighty-six percent $(346 / 401)$ of the patients had a significant decrease in their pain $(72 \%$ had complete elimination of pain and an additional $14 \%$ had a greater than $50 \%$ reduction in their pain score) by 6 months post-operatively. The procedure failed to provide pain relief in 55 patients. Median robotic microsurgical operative duration was $15 \mathrm{~min}$ (10-150 min). Complications were: one testicular ischemia, nine hematomas and two seromas. Two testicular arteries and one vasal injury occurred intra-operatively and these were repaired with robotic assisted microsurgical techniques without any further sequela. The fourth robotic arm allowed the surgeon to control one additional instrument (micro-Doppler or hydrodissector) leading to less reliance on the microsurgical assistant.

Targeted robotic assisted microsurgical denervation of the spermatic cord seems safe and feasible and the preliminary results appear promising. The four arm robotic approach allows the microsurgeon to maneuver multiple instruments simultaneously and improves operative efficiency.

\section{ROBOTIC ASSISTED MICROSURGICAL VARICOCELECTOMY}

Previous studies have shown improvement in sperm count, motility and function with the surgical treatment of a clinical varicocele. ${ }^{35,36}$ Several papers confirmed that the subinguinal microscopic varicocelectomy has superior outcomes to other techniques, such as inguinal or intra-abdominal approaches. ${ }^{37-39}$ In 2008 , Shu et al. ${ }^{8}$ described the RAVx and compared it with pure microsurgical varicocelectomy. They found that there was no significant difference in terms of operative duration between the groups (even with their initial robotic learning curve). They also noticed that robot completely eliminates tremor during the surgery, thus making the procedure subjectively easier. However, our group has shown a significant operative duration advantage as well with RAVx over microsurgical varicocelectomy in a prospective randomized control study in a canine model. ${ }^{10}$

\section{Surgical technique of RAVx}

The technique is similar to the standard microsurgical varicocelectomy. Once the spermatic cord is brought up to the surface through a $1-\mathrm{cm}$ to $2-\mathrm{cm}$ subinguinal incision, the robotic microsurgical platform is brought in (instead of the microscope) to perform the varicocelectomy procedure. The right, left and the fourth robot arms are loaded with the Black Diamond microforceps, microbipolar grasper and curved monopolar scissors, respectively. The zero degree camera lens is loaded on the robot camera arm. The VITOM camera is also positioned to provide an additional magnified view of the cord. 
RAVx begins with the incision and separation of the cremasteric muscle layer. Localization of the testicular arteries is then performed. This is achieved by utilizing either the micro-Doppler (Vascular Technology Inc., Nashua, NH, USA) or the micro-ultrasound probe (Aloka-Hitachi Ltd, Tokyo, Japan). If the Aloka micro-ultrasound probe is utilized, the image is fed directly into the surgeon console via the TilePro software (Figure 7a). All veins are ligated using 3-0 silk sutures. We utilize black and white sutures that help to identify the proximal and distal ends of the vein. After all the veins are ligated, the cord is released and the wound is closed (Figure 7b).

\section{RAVx experience}

Our group has established an institutional review board approved prospective outcomes database to follow all these patients. From June 2008 to June 2012, 181 RAVx cases were performed on 154 patients (some bilateral cases). Indications for the procedure were the presence of a grade two or three varicocele and the following conditions: oligoospermia in 60 patients, azoospermia in 17 patients and $\mathrm{CO}$ with or without oligoospermia who had failed all other conservative treatment options in 106 patients (RMDSC was also performed in many of these patients). The median duration per side was $20 \mathrm{~min}(10-80 \mathrm{~min})$. Median follow-up was 22 months (1-48 months).

Seventy-seven percent of the patients with oligoospermia had a significant improvement in sperm count or motility, $18 \%$ (three patients) with azoospermia where converted to oligoospermia and $96 \%$ of the testicular pain/orchialgia patients had a significant reduction in pain $(85 \%$ of these patients had targeted denervation of the spermatic cord in addition to varicocelectomy). Two recurrences or persistence of varicocele occurred, one patient developed a small postoperative hydrocele and two patients had post-operative scrotal hematomas (treated conservatively).

RAVx appears to be safe, feasible and efficient.

\section{ROBOTIC ASSISTED MICROTESE}

TESE is one sperm retrieval technique for patients with non-obstructive azoospermia. ${ }^{40}$ Schlege ${ }^{41}$ has shown that microTESE can improve sperm retrieval rates with minimal tissue excision. We are currently evaluating the use of robotic assistance for microTESE (ROTESE). The tri-view feature with the video link from the andrology lab microscope
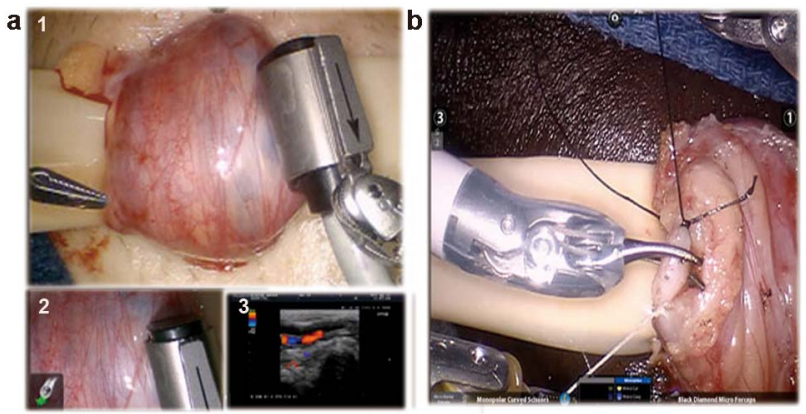

Figure 7 Surgical technique of subinguinal robotic assisted microsurgical varicocelectomy. (a) Localization of testicular vessels with Aloka real-time Doppler Ultrasound Probe (Aloka-Hitachi Ltd, Tokyo, Japan). A cockpit tri-view in the surgeon console (1) main view from the daVinci Si 3D HD camera (Intuitive Surgical, Sunnyvale, CA, USA), (2) view from the VITOM optical magnification camera lens system (Karl Storz Inc., Tuttlingen, Germany) and (3) view from the $100 \times$ optical microscopic andrology laboratory microscope (Nikon Inc., Tokyo, Japan). (b) Dilated vein is cut after being ligated with 3-0 silk ties (black and white suture ties used for convenience to identify proximal and distal ends of vein). during microTESE is a tremendous advantage for the microsurgeon. The surgeon can see the tissue being evaluated real-time and thus use this information to move to areas or tubules that appear to be more promising. This real time video communication ability while operating has improved operative efficiency for our cases.

Between July 2007 and June 2012, we have performed 12 ROTESE procedures without any complications. ROTESE is a safe and feasible procedure for sperm retrieval and subjectively appears to make tissue handling and dissection slightly easier and ergonomic compared to microTESE (Figure 8).

As better imaging modalities are developed to identify tubules that have sperm, such as multiphoton microscopy ${ }^{42}$ the robotic platform would be ideal to allow the microsurgeon to incorporate multiimaging inputs into the surgeon console.

\section{ROBOTIC ASSISTED MICROSURGICAL TESTICULAR ARTERY ANASTOMOSIS}

Our group currently has an experience of over 800 robotic microsurgery cases. However, we have had two testicular artery injuries during RMDSC procedures that were identified intra-operatively with the micro-Doppler probe. In both these cases, robotic assisted microartery repair and end-to-end anastomosis was performed using 8-0 Prolene interrupted sutures and microvascular clamps (Figure 9). Arterial flow was confirmed at the completion of the repair and both these patients have had scrotal ultrasounds (up to one year follow-up) with good testicular flow and no evidence of testicular atrophy.

\section{COST COMPARISON ANALYSIS}

One of the caveats to robotic assisted microsurgery is the increased cost of the initial purchase capital investment to acquire the robot (approximately $\$ 1.5$ million) and the annual maintenance and disposable costs (\$150000 per year). In order to create a financially viable model for this kind of program, a high volume of cases is needed to reduce the per-case cost to that comparable to pure microsurgery. The only way to justify the increased cost is by improved and enhanced surgical throughput. In our program, the use of robotic assistance has allowed the same surgeon to go from performing one or two microsurgical cases to three or four in the same time frame due to enhanced surgical efficiency and decreased surgical fatigue. It has also enabled the sur-

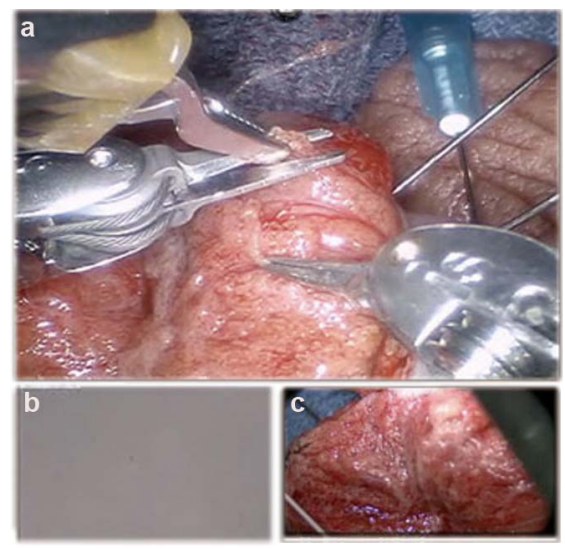

Figure 8 Robotic assisted microsurgical testicular sperm extraction. A cockpit tri-view in the surgeon console: (a) Main view from the daVinci Si 3D HD camera (Intuitive Surgical, Sunnyvale, CA, USA), (b) View from the VITOM 16-18X optical magnification camera lens system (Karl Storz Inc., Tuttlingen, Germany) and (c) View from the $100 \times$ optical micro-scopic from the andrology laboratory (Nikon Inc., Tokyo, Japan). 


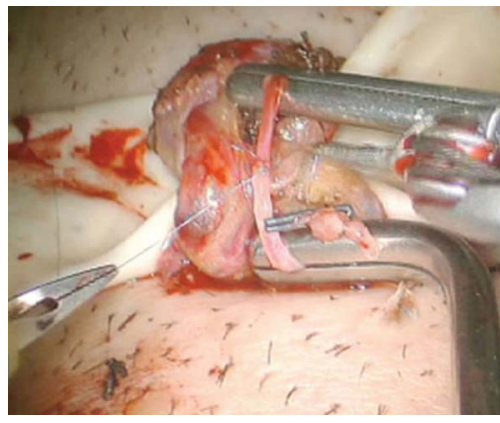

Figure 9 Robotic assisted testicular artery repair and anastomosis.

geon to run parallel rooms with minor cases in between the standard microsurgical cases, since the surgeon has decreased fatigue and tremor elimination allows enhanced surgical throughput. This model currently is only likely to be successful in high volume centers. If the price of the robotic equipment were to drop (as it likely will when other platforms become available), the use of such technology would be more universally applicable. In our center, we have been able to reduce the total out-of-pocket cost to the patient for example for the robotic vasectomy reversal to $\$ 5600$ which is $40 \%-50 \%$ lower than what our surrounding hospitals charge for a standard microscopic reversal.

\section{CONCLUSION}

The use of robotic assistance in microsurgery has been expanding not only in urology, but also in other fields such as ophthalmology, hand surgery, plastics and reconstructive surgery. The five-arm robotic approach allows the surgeon to multitask with three instruments and up to three camera views. This new platform provides the surgeon with a plethora of options and the sky is the limit in terms of what the future holds for where we could take microsurgery. The arrival of cheaper robotic systems and more types of platforms will only further expand the field. The use of adjunctive technology and fluorescence equipment with the robot may provide additional benefits in andrology. There is a multispecialty group aimed at furthering the field of robotic assisted microsurgery in a physician driven evidence-based manner-the Robotic Assisted Microsurgical and Endoscopic Society (RAMSES). This group is growing, expanding and striving to improve instrumentation, platforms and tools for robotic assisted microsurgery (www.roboticmicrosurgeons.org). There have been at least four new devices and tools developed for robotic microsurgery since the last Annual Robotic Assisted Microsurgical \& Endoscopic Society (RAMSES) meeting held in November 2011, and we hope that continued change and improvement will be the one constant.

\section{COMPETING FINANCIAL INTERESTS}

The authors declare no competing financial interests.

1 Yates DR, Vaessen C, Roupret M. From Leonardo to da Vinci: the history of robotassisted surgery in urology. BJU Int 2011; 108: 1708-13; discussion 14.

2 Gettman MT, Blute ML, Chow GK, Neururer R, Bartsch G et al. Robotic-assisted laparoscopic partial nephrectomy: technique and initial clinical experience with DaVinci robotic system. Urology 2004; 64: 914-8.

3 Menon M, Hemal AK, Tewari A, Shrivastava A, Shoma AM et al. Nerve-sparing robotassisted radical cystoprostatectomy and urinary diversion. BJU Int 2003; 92: 232-6.

4 Kuang W, Shin PR, Matin S, Thomas AJ Jr. Initial evaluation of robotic technology for microsurgical vasovasostomy. J Urol 2004; 171: 300-3.

5 Schiff J, Li PS, Goldstein M. Robotic microsurgical vasovasostomy and vasoepididymostomy: a prospective randomized study in a rat model. J Urol 2004; 171: $1720-5$.

6 Fleming C. Robot-assisted vasovasostomy. Urol Clin North Am 2004; 31: 769-72.
7 Corcione F, Esposito C, Cuccurullo D, Settembre A, Miranda N et al. Advantages and limits of robot-assisted laparoscopic surgery: preliminary experience. Surg Endosc 2005; 19: 117-9.

8 Shu T, Taghechian S, Wang R. Initial experience with robot-assisted varicocelectomy. Asian J Androl 2008; 10: 146-8.

9 Parekattil SJ, Cohen MS. Robotic surgery in male infertility and chronic orchialgia. Curr Opin Urol 2010; 20: 75-9.

10 Parekattil SJ, Brahmbhatt JV. Robotic approaches for male infertility and chronic orchialgia microsurgery. Curr Opin Urol 2011; 21: 493-9.

11 Parekattil SJ, Gudeloglu A, Brahmbhatt J, Wharton J, Priola KB. Robotic assisted versus pure microsurgical vasectomy reversal: technique and prospective database control trial. J Reconstr Microsurg 2012; 28: 435-44.

12 Sokal DC, Labrecque M. Effectiveness of vasectomy techniques. Urol Clin North Am 2009; 36: 317-29.

13 Schwingl PJ, Guess HA. Safety and effectiveness of vasectomy. Fertil Steril2000; 73 : 923-36.

14 Dohle GR, Diemer T, Kopa Z, Krausz C, Giwercman A et al. European association of urology guidelines on vasectomy. Eur Urol 2012; 61: 159-63.

15 Pile JM, Barone MA. Demographics of vasectomy — USA and international. Urol Clin North Am 2009; 36: 295-305.

16 Sandlow JI, Nagler HM. Vasectomy and vasectomy reversal: important issues. Preface. Urol Clin North Am 2009; 36: xiii-xiv.

17 Adams CE, Wald M. Risks and complications of vasectomy. Urol Clin North Am 2009; 36: 331-6.

18 O'Conor VJ. Anastomosis of vas deferens after purposeful division for sterility. J Am Med Assoc 1948; 136: 162.

19 Hulka JF, Davis JE. Vasectomy and reversible vasocclusion. Fertil Steril 1972; 23 : 683-96.

20 Silber SJ. Microsurgery in clinical urology. Urology 1975; 6: 150-3.

21 Silber SJ, Grotjan HE. Microscopic vasectomy reversal 30 years later: a summary of 4010 cases by the same surgeon. J Androl 2004; 25: 845-59.

22 Practice Committee of American Society for Reproductive Medicine. Vasectomy reversal. Fertil Steril 2008; 90 (5 Suppl): S78-82.

23 Naughton CK, Thomas AJ, Jr. Optimizing laboratory use of human vas deferens specimens for microsurgical practice. Urology 2002; 60: 320-3.

24 Costabile RA, Hahn M, McLeod DG. Chronic orchialgia in the pain prone patient: the clinical perspective. J Urol 1991; 146: 1571-4.

25 Davis BE, Noble MJ, Weigel JW, Foret JD, Mebust WK. Analysis and management of chronic testicular pain. J Urol 1990; 143: 936-9.

26 McMahon AJ, Buckley J, Taylor A, Lloyd SN, Deane RF et al. Chronic testicular pain following vasectomy. Br J Urol 1992; 69: 188-91.

27 Alfieri S, Amid PK, Campanelli G, Izard G, Kehlet H et al. International guidelines for prevention and management of post-operative chronic pain following inguinal hernia surgery. Hernia 2011; 15: 239-49.

28 Levine LA. Microsurgical denervation of the spermatic cord. J Sex Med 2008; 5: 5269.

29 Strom KH, Levine LA. Microsurgical denervation of the spermatic cord for chronic orchialgia: long-term results from a single center. J Urol 2008; 180: 949-53.

30 Thacker MA, Clark AK, Marchand F, McMahon SB. Pathophysiology of peripheral neuropathic pain: immune cells and molecules. Anesth Analg 2007; 105: 838-47.

31 Parekattil S, Priola K, Atalah H, Cohen M, Vieweg J et al. Trifecta of Pain: Anatomic Basis for Denervation of the Spermatic Cord for Chronic Orchialgia. J Urol 2010; 183: e730-1.

32 Devine CJ Jr., Schellhammer PF. The use of microsurgical denervation of the spermatic cord for orchialgia. Trans Am Assoc Genito-Urin Surg 1978; 70: 149-51.

33 Gudeloglu A, Iqbal Z, Parekattil SJ, Groth AC, Priola KB et al. Hydrodissection for improved microsurgical denervation of the spermatic cord: prospective blinded randomized control trial In a rat model. Fertil Steril 2011; 96: S87-8.

34 Parekattil SJ, Gudeloglu A, Brahmbhatt J, Priola KB, Cohen MS. Prospective randomized control trial of a neuroprotective wrap for the spermatic cord after denervation for chronic orchialgia. Fertil Steril 2011; 96: S231.

35 Schauer I, Madersbacher S, Jost R, Hubner WA, Imhof M. The impact of varicocelectomy on sperm parameters: a meta-analysis. J Urol 2012; 187: 1540-7.

36 Gabriel MS, Chan SW, Alhathal N, Chen JZ, Zini A. Influence of microsurgical varicocelectomy on human sperm mitochondrial DNA copy number: a pilot study. $J$ Assist Reprod Gen 2012; 29: 759-64.

37 Chen XF, Zhou LX, Liu YD, Ping P, Chen YH et al. Comparative analysis of three different surgical approaches to varicocelectomy. Zhonghua Nan Ke Xue 2009; 15: 413-6. Chinese.

38 Abdel-Maguid AF, Othman I. Microsurgical and nonmagnified subinguinal varicocelectomy for infertile men: a comparative study. Fertil Steril 2010; 94: 2600-3.

39 Ding H, Tian J, Du W, Zhang L, Wang $\mathrm{H}$ et al. Open non-microsurgical, laparoscopic or open microsurgical varicocelectomy for male infertility: a meta-analysis of randomized controlled trials. BJU Int 2012; 110: 1536-42.

40 Colpi GM, Piediferro G, Nerva F, Giacchetta D, Colpi EM et al. Sperm retrieval for intracytoplasmic sperm injection in non-obstructive azoospermia. Minerva Urol Nefrol 2005; 57: 99-107.

41 Schlegel PN. Testicular sperm extraction: microdissection improves sperm yield with minimal tissue excision. Hum Reprod 1999; 14: 131-5.

42 Najari BB, Ramasamy R, Sterling J, Aggarwal A, Sheth S et al. Pilot study of the correlation of multiphoton tomography of ex vivo human testis with histology. J Urol 2012; 188: 538-43. 